\title{
Simultaneous use of multiple seismic arrays
}

\author{
J. Stipčević, ${ }^{1,2}$ B.L.N. Kennett ${ }^{1}$ and H. Tkalčić ${ }^{1}$ \\ ${ }^{1}$ Research School of Earth Sciences, The Australian National University, Canberra ACT 2601, Australia. E-mail: jstipcevic@gfz.hr \\ ${ }^{2}$ Department of Geophysics, Faculty of Science, University of Zagreb, 10000 Zagreb, Croatia
}

Accepted 2017 January 20. Received 2017 January 19; in original form 2015 October 16

\begin{abstract}
S U M M A R Y
Seismic arrays provide an important means of enhancing seismic signals and determining the directional properties of the wavefield by beamforming. When multiple arrays are to be used together, the viewpoint needs to be modified from looking outwards from each array to focusing on a specific target area and so constraining the portions of the waveforms to be analysed. Beamforming for each array is supplemented by the relative time constraints for propagation from the target to each array to provide tight spatial control. Simultaneous multiple array analysis provides a powerful tool for source characterization, and for structural analysis of scatterers as virtual sources. The multiple array concept allows us to illuminate a specific point in the Earth from many different directions and thus maps detailed patterns of heterogeneity in the Earth. Furthermore, illumination of the structure from multiple directions using data from the same event minimizes source effects to provide clearer images of heterogeneity. The analysis is based on a similar concept to the backprojection technique, where a part of the seismic wave train is mapped to a specific point in space by ray tracing. In contrast to classic backprojection where the incoming energy is mapped onto a horizontal plane with limited vertical resolution, the multiarray method controls depth response by combining relative time constraints between the arrays and conventional beamforming. We illustrate this approach with application to two earthquakes at moderate depth. The results show that the use of simultaneous multiple arrays can provide improvement both in signal quality and resolution, with the additional benefit of being able to accurately locate the source of the incoming energy and map large areas with only a limited number of such arrays.
\end{abstract}

Key words: Spatial analysis; Earthquake source observations; Body waves.

\section{INTRODUCTION}

Seismic arrays play an important role in the detection and location of seismic events. The number of arrays of various types has been augmented in recent years by the new arrays in the primary seismic network established by the Preparatory Commission for the Comprehensive Nuclear-Test-Ban Treaty (CTBT) with the aim of securing more uniform detection capabilities across the globe. Other new arrays, such as Pilbara Seismic Array (PSAR) in northwestern Australia form part of enhanced tsunami warning systems.

The merit of seismic arrays is that when the signals at the various sensors are suitably phased, coherent signal is enhanced relative to incoherent background noise (e.g. Rost \& Thomas 2002); the theoretical gain factor in the stacked energy for $N$ sensors is $N$. For a single seismic array, the natural mode of operation is to look outwards from the array with monitoring of the full range of slowness and azimuths seeking to detect new events. Detections from multiple arrays can then be brought together to refine the location of the event, along with information from other stations.
In this paper, we examine the way in which multiple seismic arrays can be used simultaneously to look at seismic sources or the location of seismic scattering, which can be regarded as virtual sources. Such simultaneous use of multiple arrays requires concentration on those portions of the seismograms related to the same potential event, with appropriate time delays to compensate for the different epicentral distances to the arrays. Thus rather than looking outwards from a single array and sweeping through the full range of distances and azimuths via beamforming, multiarray analysis is automatically source oriented.

This difference in approach has been recognized implicitly in the use of multiple arrays to track the spatial evolution of great earthquakes. Thus Roessler et al. (2010) have exploited the coherence properties of seismograms recorded at networks of stations, treated as very large arrays, as a function of apparent source position in the neighbourhood of the hypocentre of large earthquakes. Rather than summing the semblance responses for the different large arrays they have multiplied them, which gives higher spatial resolution. Many backprojection studies for great earthquakes have employed more than one suite of stations as an array, but commonly have just 
compared the results from the different receiver locations (e.g. Simons et al. 2011; Ye et al. 2013). Kiser \& Ishii (2012) have demonstrated the improvement that can be made when two arrays at different distances and azimuths are used together through simulations of the combined response of the central portion of the US array and the Hi-net network in Japan.

We here show how the combination of the slowness and time constraints from multiple seismic arrays can be used to achieve good spatial resolution in 3-D, when there is sufficient azimuthal separation between the arrays employed. We illustrate the simultaneous analysis by using the set of arrays within Australia to examine events in the subduction zones to the north, and also show the improvement achieved when additional azimuths are brought to bear by using North American arrays.

\section{SINGLE ARRAY RESPONSE}

At a moderate aperture array, the seismic wave front impinging on the group of sensors from a distant event can be approximated by a plane wave, with vector slowness

$\mathbf{s}=\left(s_{1}, s_{2}, s_{3}\right)=s(\cos \varphi \sin i, \sin \varphi \sin i, \cos i)$,

where $i$ is the inclination to the vertical and $\varphi$ is the azimuth from north. The coordinates $s_{1}, s_{2}$ and $s_{3}$ represent the north, east and vertical directions. Although we define slowness through inclination angle, we are only able to detect the horizontal slowness $\{s \sin i\}$ of the plane wave. This means that conventional array methods are good for determining the direction of the incoming energy, but will not be able to determine the absolute location of the energy source.

For sensor coordinates $\left\{\mathbf{x}_{j}=\left(x_{j 1}, x_{j 2}, x_{j 3}\right)\right\}$ relative to a reference site at the origin, the wave front delay times across the array $\left\{t_{j}\right\}$ are determined by the projection of the slowness onto the coordinate shifts.

For frequency $\omega$, the plane wave front produces a pattern of phase delays across the array sensors:

$\exp \left[\mathrm{i} \omega t_{j}\left(\mathbf{x}_{j}, \mathbf{s}\right)\right]=\exp \left[\mathrm{i} \omega\left(s_{1} x_{j 1}+s_{2} x_{j 2}+s_{3} x_{j 3}\right]\right.$.

Unless the array has unusually high topographic relief which is not the case with the small arrays used in this research, the vertical contribution $s_{3} x_{j 3}$ is not usually significant.

The properties of the seismic array can then be characterized by the array response function

$\mathcal{S}(\Delta \mathbf{s}, \omega)=\frac{1}{N} \sum_{j=1}^{N} \exp \left\{\mathrm{i} \omega\left[\delta \mathbf{s} . \mathbf{x}_{J}\right]\right\}$

for differential slowness $\delta \mathbf{s}$, which represents a scaled version of the Fourier transform, with respect to wavenumber, of a set of delta functions placed at the sensor locations. The main peak is at $\delta \mathbf{s}=0$, but subsidiary peaks can arise from the specific geometry of the array. The beam peak is tighter for a larger array, provided the signals remain coherent.

For a simple linear stack of outputs from the sensors phased to a specified slowness, the array response $\mathcal{S}$ modulates the seismic wavefield in the slowness domain (see e.g. Kennett 2002, Chapter 23). For each incident plane wave, the same characteristic pattern of array response is present though shifted to be centred on the incoming slowness of the plane wave front. More sophisticated analysis with nonlinear processing can tighten the determination of slowness estimates from observations (e.g. Gal et al. 2014), but the basic geometrical constraints remain.
With a specified vector slowness an array beam can be related to potential geographic locations via the slowness translated to epicentral distance and the azimuth. The relatively slow variations in slowness with epicentral distance and the width of the main lobe means that a considerable geographic region is included at the Earth's surface.

\section{COMBINING MULTIPLE ARRAYS}

When working with multiple arrays we can still make use of beamforming at each of the arrays. To bring the information to bear on a common object, the analysis has to be carried out on appropriate time segments of the records.

In retrospective analysis, the various arrays can be used separately with optimum stacking for the target source. Such an approach has often been used in discussion of the character of seismic sources in forensic seismology (e.g. Bowers \& Selby 2009; Douglas 2013). In contrast, when we seek to exploit multiple arrays simultaneously we no longer look outward from each of the arrays with a slowness beam analysis. Instead we have to select a target point and determine the time of passage from that point to each of the array reference points (usually the centre). This then imposes specific time delays for the target which have to be applied to the different array beams before they can be combined. The array beams have to be constructed for the appropriate slowness associated with the epicentral distances and positions of each array relative to the target. The final result can be a composite stack, either linear or with coherence enhancement applied at each of the individual arrays.

The use of spatial rather than slowness oriented analysis is familiar in efforts to track the time evolution of major seismic events using large networks of seismic stations. Yet, commonly analysis is conducted for separate configurations of stations (Simons et al. 2011; Ye et al. 2013) and there are few studies employing multiple networks. Kiser \& Ishii (2012) have demonstrated the potential benefit of using North American and Japanese stations together in a study exploring several subduction zone earthquakes. The work of Roessler et al. (2010) employed several groups of widely distributed seismic stations as an array, examining the coherence of array stacks as a function of spatial position.

Here we show how multiple modest size arrays can be used simultaneously, exploiting slowness beamforming guided by a spatial grid with array alignment to select portions of the seismograms that bear on the same source characteristics. In the examples of multiple array analysis below we use six open-access seismic arrays with a range of apertures and station configuration. We employ four arrays in Australia (ASAR, PSAR, WRA and SQspa) and two in North America (ILAR and YKA). The arrays consist of between 13 and 26 sensors (both broad-band and short period) with apertures ranging from 10 to $25 \mathrm{~km}$. The configurations of these arrays are illustrated in Fig. 1. The small/medium aperture of the arrays allows for the coherency of high frequency signals at all components. In the Supporting Information (Fig. S1) we show the array responses for all of these arrays.

The Alice Springs Array (ASAR) in the Northern Territory of Australia is a long established array with a modest aperture of about $12 \mathrm{~km}$ and a cluster of 19 sensor elements. The Warramunga Array (WRA) also in the Northern Territory, about $500 \mathrm{~km}$ north of Alice Springs, is L-shaped with a $25 \mathrm{~km}$ aperture. WRA has been operating since 1968; upgrade to CTBT standards in 1999 introduced 4 further stations near the crossing of the arms to give 24 stations in total. The PSAR in northwestern Australia has 13 stations 

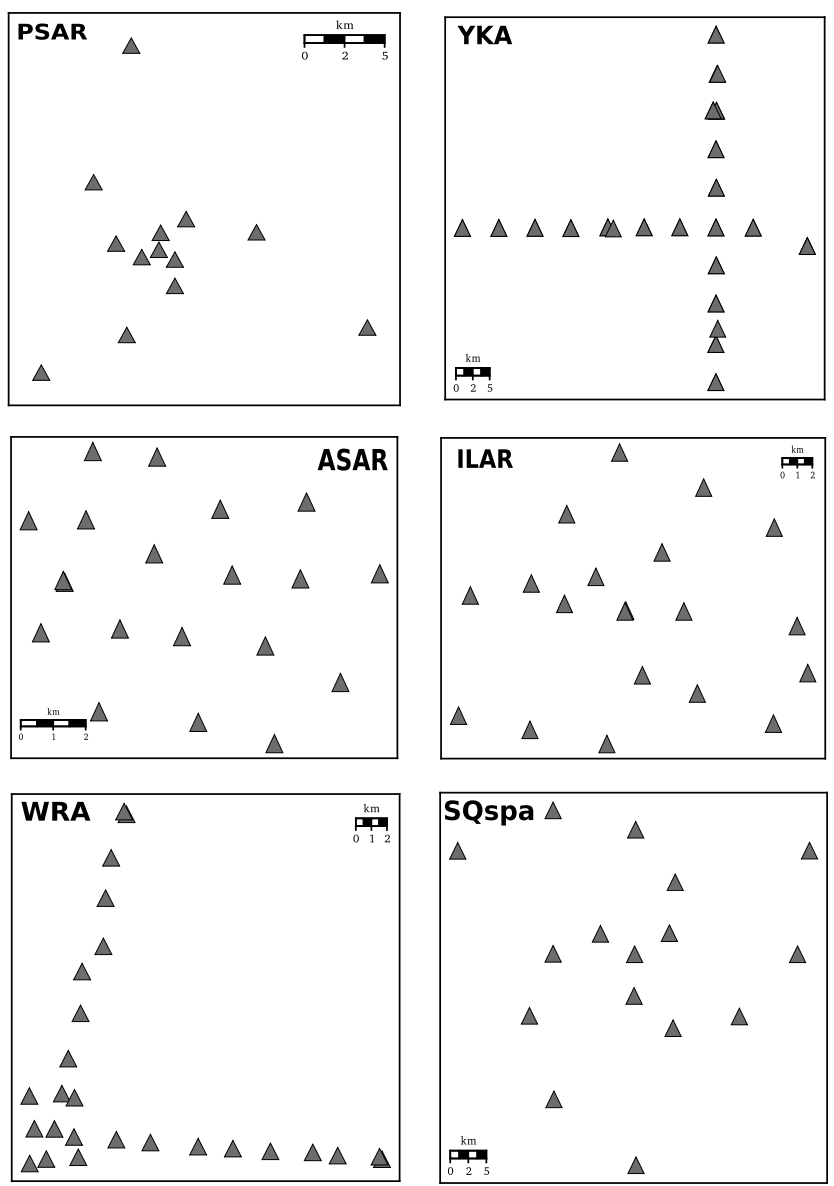

Figure 1. Configuration of the six arrays used for multiple array analysis.

along three spiral arms with logarithmic spacing with a $13 \mathrm{~km}$ aperture. The temporary SQspa array in southern Queensland has 16 stations employed along three spiral arms with regular ring spacing, with the outer ring at $25 \mathrm{~km}$ from the centre. The Yellowknife array (YKA) in Canada has an aperture of $25 \mathrm{~km}$ and comprises a cross with 18 sensors and four additional broad-band seismometers. ILAR in Alaska is a cluster array of aperture about $12 \mathrm{~km}$ and 19 sensor elements.

We will first consider the nature of the spatial resolution that can be achieved with multiple arrays, and in the early illustrations will concentrate attention on the use of just three arrays so that the individual contributions can be readily identified by colour. Further we present several synthetic tests investigating the lateral and depth resolution from the combined use of the six arrays. We then move our attention to the analysis of two intermediate depth events in the subduction zones east and west of Australia and show how our approach fares in the real case scenario, that is, how different factors such as scattering, time correction and azimuthal coverage influence the final results.

\section{SPATIAL RESOLUTION WITH MULTIPLE ARRAYS}

What is the expected spatial resolution associated with a multiple array stack? We examine the contributions from the array response in slowness and the way that these relate to spatial dependence. We then show the way in which time constraints serve to concentrate the spatial dependence. The combination of slowness response and time constraints serves to concentrate on a spatial target.

Consider a target location $\mathbf{X}_{0}$ and an array at an epicentral distance $\Delta_{0}$ and azimuth $\varphi_{0}$ to the reference point, for which the traveltime for the relevant seismic phase is $T$.

\subsection{Slowness constraints}

Based on an appropriate earth-model we can construct the expected slowness $s_{0}$ for waves emitted from the target location arriving at the reference point of the array. For positions displaced from the target the slowness will change, and the response in spherical slowness about $s_{0}$ will be modulated by the array response function $\mathcal{S}(\delta \mathbf{s}, \omega)$, where $\delta \mathbf{s}$ is the deviation in vector slowness from $s_{0}$. The array response function will in turn be mapped back into an effective spatial response around the target. Effective spatial response is the area around the target that we can resolve and the size of this area depends on the array geometry and the frequency content of the signal.

In the immediate vicinity of the target point $\mathbf{X}_{0}$, attention will be focussed on the main peak in the array response. Suppose now we move the source target point from $\mathbf{X}_{0}$ to $\mathbf{X}_{0}+\mathbf{x}$. Then expanding the expression for the relation between the changes in vector slowness $\mathbf{s}$ and spatial displacement $\mathbf{x}$ from the target point to first order in $\mathbf{x}$ :

$\mathbf{s}=\mathbf{s}_{0}+\left.\frac{\partial \mathbf{s}}{\partial \mathbf{x}}\right|_{\mathbf{s}_{0}} \mathbf{x}+\mathcal{O}\left(\mathbf{x}^{2}\right)$.

Using the chain rule for differentiation, we can express (4) in terms of the epicentral distance $\Delta$,

$\delta \mathbf{s}=\frac{\partial \mathbf{s}}{\partial \mathbf{x}} \mathbf{x}=\frac{\partial \mathbf{s}}{\partial \Delta} \cdot \frac{\partial \Delta}{\partial \mathbf{x}} \mathbf{x}$.

In a isotropic 1-D earth model, the original vector slowness $\mathbf{s}_{0}$, and the gradient of epicentral distance link to the unit vector $\mathbf{e}_{r}$ along the back azimuth direction,

$\mathbf{s}_{0}=s_{0} \mathbf{e}_{r} ; \quad \frac{\partial \Delta}{\partial \mathbf{x}}=-\mathbf{e}_{r} ;$

since there is no variation in epicentral distance $\Delta$ perpendicular to the great-circle path. Thus we can recast (5) as

$\delta \mathbf{s}=-\frac{\partial s}{\partial \Delta}\left(\mathbf{x} . \mathbf{e}_{r}\right) \mathbf{e}_{r}$

involving a tensor rotation with respect to azimuth. Explicitly for a horizontal displacement $\mathbf{x}=\left[\delta x_{1}, \delta x_{2}\right], \mathbf{e}_{r}=[\sin \varphi, \cos \varphi]$, where $\varphi$ is azimuth from north, the slowness increments are

$\left[\delta s_{1}, \delta s_{2}\right]=-\frac{\partial s}{\partial \Delta}\left(\delta x_{1} \sin \varphi+\delta x_{2} \cos \varphi\right)[\sin \varphi, \cos \varphi]$.

The peak of the array response in space is therefore scaled by the inverse of the rate of change of slowness with distance and skewed by the azimuth dependent tensor rotation. This will give an effective elongation of the response along the great-circle between target and array (Fig. 2).

For teleseismic $P$ waves, $\partial s / \partial \Delta$ is negative and slowly varying so similar spatial scaling will apply to multiple arrays. The spatial response from the combination linear stacks for multiple arrays in the immediate neighbourhood of the target location will therefore resemble a scaled sum of the array responses for the individual arrays. The effects of the different tensor rotations will be more evident when the arrays span a large range of azimuths. 


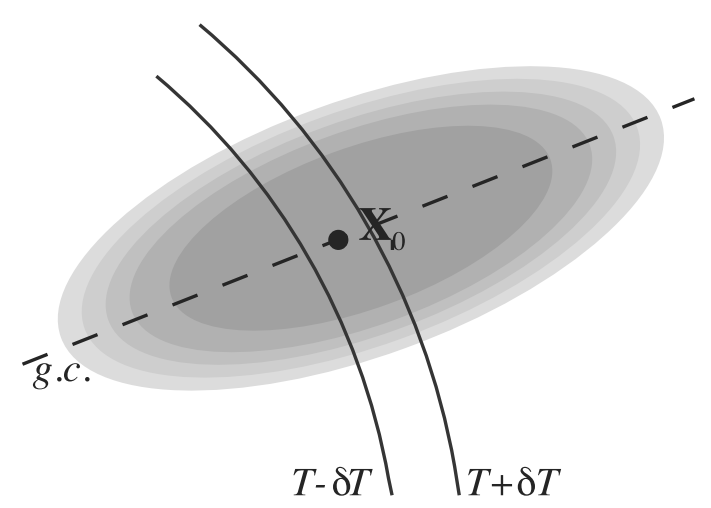

Figure 2. Schematic representation of the interaction of slowness and time constraints for a single array in the neighbourhood of a target point $\mathbf{X}_{0}$. The slowness peak is elongated along the great circle (g.c.), while the time band cuts perpendicularly.

\subsection{Time constraints}

To look at the way in which the relative timing at the different arrays acts to affect spatial resolution we can build on the analysis developed by Kennett et al. (2014) for multistation energy projection to determine the character of seismic sources.

Consider a single seismic phase, such as $\mathrm{P}$, emitted from the target location $\left(\mathbf{X}_{0}\right)$ arriving at the reference point of the array. For an array with a central point location $\mathbf{X}_{j}$ with epicentral distance $\Delta_{j}$ from a target location $\mathbf{X}_{0}$, the time of passage for a chosen phase is

$t_{j}\left(\mathbf{X}_{j}, \mathbf{X}_{0}\right)=T_{j}\left(\Delta_{j}\right)=T_{j}^{0}\left(\Delta_{j}\right)+\delta T_{j}\left(\Delta_{j}\right)$,

where $T_{j}^{0}\left(\Delta_{j}\right)$ is the time estimated from a reference earth model. $\delta T_{j}\left(\Delta_{j}\right)$ is a local, array-specific, correction depending on the spherical slowness $s_{j}$ via $\Delta_{j}$.

Suppose now we move the source target point from $\mathbf{X}_{0}$ to $\mathbf{X}_{0}+\mathbf{x}$. Then the expanding expression for the perturbed time to first order in $\mathbf{x}$ :

$$
\begin{aligned}
& t_{j}\left(\mathbf{X}_{j}, \mathbf{X}_{0}+\mathbf{x}\right) \\
& =T_{j}\left(\Delta_{j}\right)+\frac{\partial T_{j}}{\partial \mathbf{x}} \cdot \mathbf{x}+\mathcal{O}\left(\mathbf{x}^{2}\right) \\
& =T_{j}\left(\Delta_{j}\right)+\frac{\partial T_{j}}{\partial \Delta_{j}} \frac{\partial \Delta_{j}}{\partial \mathbf{x}} \cdot \mathbf{x}+\mathcal{O}\left(\mathbf{x}^{2}\right) \\
& =T_{j}^{0}\left(\Delta_{j}\right)+s_{j} \frac{\partial \Delta_{j}}{\partial \mathbf{x}} \cdot \mathbf{x}+\mathcal{O}\left(\mathbf{x}^{2}\right)
\end{aligned}
$$

since the slowness $s_{j}=\partial T_{j}^{0} / \partial \Delta_{j}$ for the phase arrival at central point of the array $j$. For spatial displacement $\mathbf{x}$ that follows the isochron $t_{j}\left(\mathbf{X}_{j}, \mathbf{X}_{0}\right)=T_{j}\left(\Delta_{j}\right)$ there is no change in traveltime and $\partial T_{j} / \partial \mathbf{x}=0$. This stationary component represents the dominant influence in the neighbourhood of the target point in three dimensions. For a 1-D earth model the projection of this stationary component onto the Earth's surface would correspond to a circle on a sphere with a centre at $\mathbf{X}_{j}$ and radius $\Delta_{j}$ passing thorough the target point $\mathbf{X}_{0}$.

Consider now a time window $\Delta T$ around the expected phase arrival time $T$. According to the derivation above this time window will map into a spatial zone around the locus of the isochron of width $\Delta T / s_{0}$. For an isotropic and laterally homogeneous earth this zone around the isochron will cut the great-circle path at right angles. For a 1-D Earth model the projection of the time window in space onto the Earth's surface would correspond to a band around a small circle on the sphere drawn with centre at the array and radius $\Delta_{0}$ passing through the target point $\mathbf{X}_{0}$ (Fig. 2). The plump isochron concept works just as well for a model incorporating the 3-D structure of the Earth, but then the geometric shape at the surface would deviate somewhat from the small circle.

A time segment for a close array maps to an influence zone with a tight radius of curvature and a narrow band about the isochron since the slowness $s_{0}$ is large, and so can be very important for improving resolution (for details see Kennett et al. 2014).

\subsection{Combined slowness and time constraints for multiarrays}

Our aim in this section is to combine the best from beamforming and backprojection processes in order to get an estimate of high frequency energy in space. We build on slowness and time restrictions described in Sections 4.1 and 4.2. Whereas the beamforming will mostly be influenced by the changes in slowness the backprojection will mostly rely on the traveltime from the target location (Fig. 2).

We can describe the process as follows: consider an event with hypocentre at location $\mathbf{x}_{h}$ and origin time $t_{0}$. For a source at $\mathbf{x}_{k}$ and initiation time $t_{0}+T$ calculate the traveltime $t_{j k}$ and slowness $s_{j k}$ for a phase at the central point of the array $\left\{\mathbf{x}_{j}\right\}$. For each array $\left\{\mathbf{x}_{j}\right\}$ construct an estimator for the radiated energy associated with this location and origin time by constructing an energy estimate over time interval $\delta t$. Energy estimate for each array is formed by first creating a beam (delay and sum) from all the traces in the array $\left\{u_{i j}\right\}$ based on the slowness $\mathbf{s}_{j k}$ and then summing over time interval $\delta t$,

$E(T)_{j k}=\int_{0}^{\delta t} w(\tau) \sum_{i=1}^{N} u_{i j}^{2}\left(T+t_{j k}+\mathbf{r}_{i} \mathbf{s}_{j k}\right) d \tau$

with the coordinate vector $\mathbf{r}_{i}$ for each of $N$ array stations and window function $w(\tau)$. The offset time $T$ is initially set as 0 but can be progressively incremented for larger earthquakes with longer duration. In this way we can track progression of the highest energy emission in space.

Due to the limited resolving power of the individual medium sized array we can combine energy estimates of several arrays to get a well resolved source area. We can combine the individual array estimates by either pointwise summation or multiplication.

$E(T)=\sum_{j=1}^{M} E(T)_{j} \quad$ or $\quad E(T)=\prod_{j=1}^{M} E(T)_{j}$,

where $M$ is the number of the arrays used. Later we show that for most cases multiplication leads to more distinct central lobe and suppresses unwanted noise (see also Roessler et al. 2010).

We now move to illustrate the way in which the different aspects of the spatial response for multiple arrays interact with an example for a target placed beneath the Solomon Islands at $200 \mathrm{~km}$ depth, using three of the arrays in Australia. We use the 1-D reference model ak135 (Kennett et al. 1995) to calculate traveltimes and slownesses.

We show the behaviour from the use of multiple arrays in 3-D with intersecting horizontal and vertical cuts through the target. The response for each of the three arrays (PASR, WRA and SQspa) is assigned to a separate colour channel and then plotted for each voxel (or the section in the relevant plane). In Fig. 3, we have placed PSAR on the magenta channel, WRA on the cyan channel and SQspa on a 


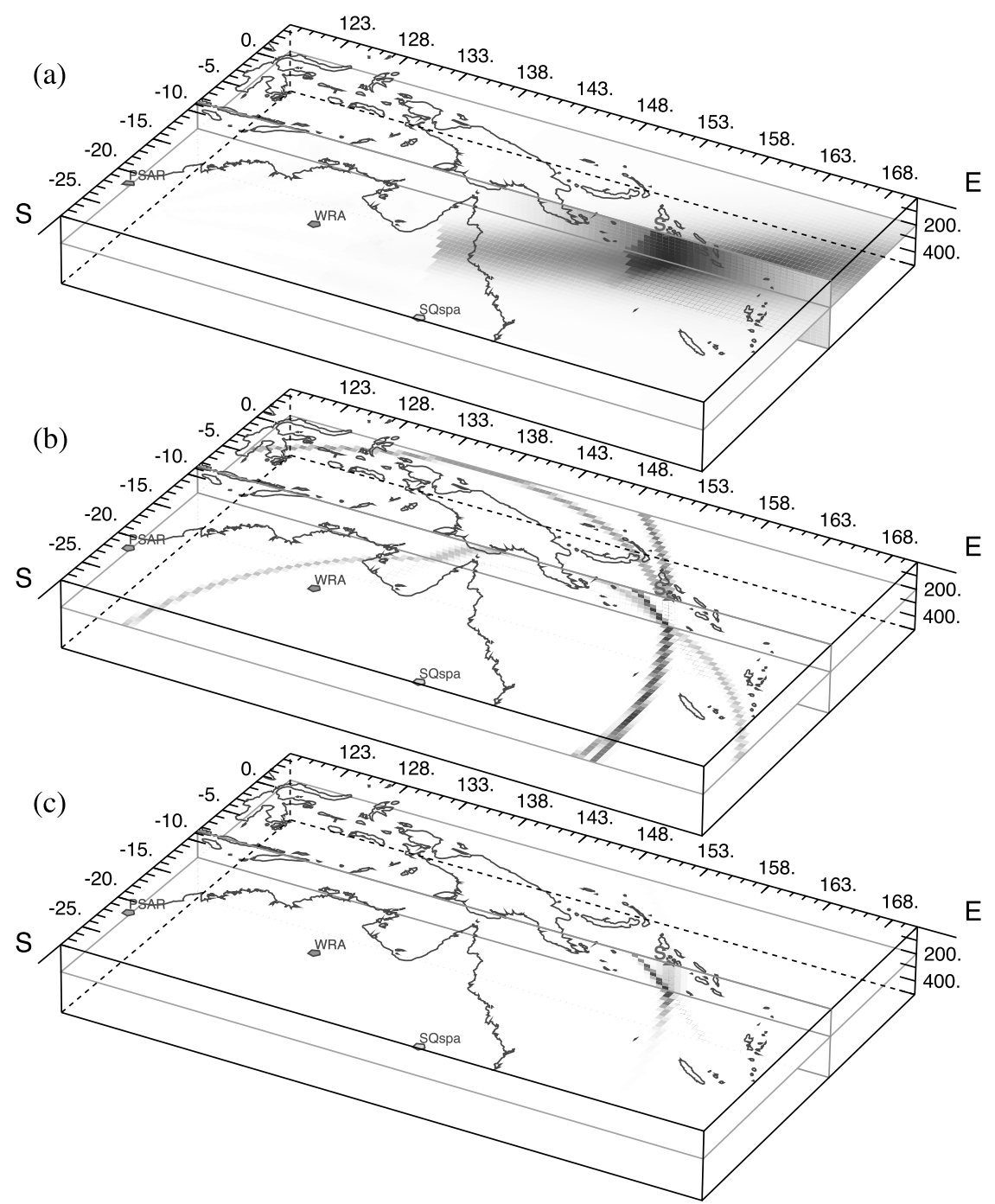

Figure 3. Illustration of the interaction of spatial constraints for multiple arrays target with a target beneath the Solomon Islands at a depth of $200 \mathrm{~km}$. The surface projection of the target is shown by a purple triangle marked with S. The contributions from the three arrays are shown in different colours, PSAR in Western Australia in magenta, WRA in the Northern Territory in cyan and SQspa in South Queensland in light green, so that the combined full-strength contribution is almost black. Intersecting vertical and horizontal planes through the target are shown: (a) array response in slowness mapped into space for the target slowness; (b) time constraints for a $4 \mathrm{~s}$ band around the P isochron passing through the target point; (c) The combined effect of multiplying the slowness and time response with strong concentration at the target. The slownesses and traveltimes for each $0.5^{\circ} \times 0.5^{\circ} \times 25 \mathrm{~km}$ voxel were calculated at the cell centre using the ak135 model.

light green channel, so that full-strength response will combine to a near black.

The three arrays are focussed at the target point beneath the Solomons. We map the array responses $\mathcal{S}$ on to space by calculating the vector slowness at voxel centres spaced at $0.5^{\circ} \times 0.5^{\circ} \times 25 \mathrm{~km}$ and then extracting the appropriate contribution from the array response $\mathcal{S}$, with normalization to unity at the slowness target. The combined energy response of the three arrays in slowness is shown in 3(a). PSAR has the smallest aperture and hence the largest width to the main peak in slowness. When coupled with the slow change in slowness with epicentral distance, the result is a broad spatial sampling in magenta even though the beam is targeted. The larger aperture of the WRA array gives a more constrained array response (in cyan), but still distributed along and around the great circle to the target. The combination of the contributions from PSAR and WRA appears as a dark purple. The SQspa array has the largest aperture and the closest distance. This results in a well confined array re- sponse in the horizontal plane. However, there is little variation of slowness with depth of source down to $200 \mathrm{~km}$ for the ak135 model and so we see a columnar dark grey structure reaching up from the true target towards the surface. The linear combination of the three arrays gives good positional control, but weak depth constraints.

With the three arrays directed at the target, we impose appropriate time delays so that the portions of the seismogram for the same seismic phase are brought to bear simultaneously. In Fig. 3(b), we illustrate $4 \mathrm{~s}$ bands about the isochron for $P$-waves from a source at the target to each of the arrays, using the same colour scheme as in Fig. 3(a).

The size of the window is chosen on the basis of the frequency content of the signal. The frequency content of the seismogram and size of the window coalesce to form the effective spatial resolution (Kennett et al. 2014).

The paths of the plump isochrons represent small circles on a horizontal plane, but show varying curvature in depth thanks to 
the variation in seismic wave speeds. Although the azimuthal span between the three arrays is small, there is a limited zone in which all three isochronal constraints are satisfied. There is considerable overlap between the isochronal bands from PSAR and WRA because of their similar azimuths from the target. The green band from SQspa cuts across the others to restrict the zone in both the horizontal and vertical planes.

The full spatial resolution achievable with the multiple array analysis can then be extracted by combining the slowness and time constraints as in Fig. 3(c). For each array we have multiplied the slowness response in (a) by the isochronal band in (b) and then superimposed the results. The combined response is tightly focussed at the target, with limited leakage associated with the contributions from the individual arrays. We see the residual effect of the columnar slowness feature from SQspa, and for PSAR and WRA the main contributions come from the region of overlap of the isochronal bands. Even with the not very favourable geometry of the three arrays relative to the target, good spatial localization is attainable in the neighbourhood of the target, so that discrimination is possible for nearby sources even with simple linear stacking at each array. For the case illustrated in Fig. 3 there would be no difficulty in separately resolving sources $100 \mathrm{~km}$ away from the original target.

The style of analysis we have illustrated in Fig. 3 can be readily adapted to the overall response from many arrays, the restriction to just three arrays enabled us to isolate the individual contributions. It is therefore possible to investigate the spatial resolution attainable for any source zone of interest, or for seismic scatter configurations that may occur outside the normal depth range of earthquakes.

\subsection{Lateral and depth resolution}

Here we build upon the example shown in the previous section and create synthetic seismic traces which are then used in simultaneous array analysis, using the same procedures as are employed for the real data events in Section 5. In this way we explore lateral and depth resolution for target mapping using the six arrays (ASAR, PSAR, WRA, SQspa, ILAR and YKA). Synthetic seismograms are generated using the Ricker wavelet (Ricker 1953) with central frequency of $1 \mathrm{~Hz}$. The traveltimes and slownesses are determined using the 1-D reference model akl35 (Kennett et al. 1995) for a given source location. Random noise is added to the synthetic seismograms with signal-to-noise ratio of 10 .

We start by creating a simple source in the area of the Solomon Islands. The source location was chosen to match the location of the real earthquake used in the first example in the next section. The depth of the source was set to $100 \mathrm{~km}$. In the upper six panels of the Fig. 4 we show the beam-power contributions from each of the arrays separately. The two distant North American arrays (ILAR and YKA) provide very limited directional constraints but have excellent control on the source distance. The other four arrays give much better directional control with larger-aperture arrays (SQspa and WRA) more effective than the two smaller-aperture arrays (ASAR and PSAR). The results for the array SQspa are particularly intriguing, since the spiral-arm configuration (Kennett et al. 2015) gives excellent directional control. The two lowermost panels in Fig. 4 show the lateral resolution when contributions from all six arrays are combined. The left panel shows the results with coarser grid spacing and the right panel displays results on a finer grid. We have used summation of beam-power maps from the individual arrays in order to show leakage away from the target location. Alternatively, we can use pointwise multiplication on the grid for all the separate array contributions to produce a composite response in which all the artefacts would be suppressed (Fig. 5). As this synthetic test shows, with the six array configuration lateral resolution is very good, and the area of the highest beam power is tightly focused around the source.

The results for resolution in depth are illustrated in Fig. 5. Once again the area of the highest beam power is focused around the true source location, although with some depth smearing in the longitudinal direction due to the stronger influence of the Australian arrays. Nevertheless, our results show that even with this limited array configuration we can expect depth resolution of approximately $\pm 10 \mathrm{~km}$. For comparison, in the two lower panels, we show the results with the use of pointwise multiplication of the array beam-power contributions. The source location is now much tightly constrained and all the artefacts are subdued. The depth resolution would improve dramatically with the addition of at least one more array well spaced in azimuth. Also, as shown by Kiser et al. (2011), depth resolution can be improved by using more seismic phases such as $\mathrm{pP}$ or sP.

In Fig. 6, we show an enlarged area around the source area for the example in Solomon Islands. The results suggest that with this configuration around the source area we have potential resolution of about $30 \mathrm{~km}$. The spatial resolution for the multiarray configuration is given by the sum (or multiplication) of the spatial contributions from neighbourhood of the isochrons passing through the source target point. The slowness part allows for the identification of the region in which an event has occurred. When both time and slowness are used in search mode around an event location, they have the effect of honing the stacking of the individual arrays and the effective resolution from the array geometry is based on coalescence of time windows.

Full spatial resolution for the procedure presented in this manuscript consists of two main contributors, the frequency content of the signal (e.g. the dominant frequency) and the window size used (Kennett et al. 2014). Our main goal in this work is to show that by using small to medium sized array we are able to extract higher frequency content. This is true in most cases for smaller arrays as signal will be coherent even at high frequencies for close enough stations. By beamforming seismograms filtered at higher corner frequencies we can in principle get better resolution at the source area. Therefore, window size has to match frequency content in order to get better resolution as too large (or too small) a window would artificially smear the effective resolutions (or artificially enhance it for too small window).

\subsection{Multiple sources}

In addition to determining the potential resolution for a single point source, it is important to know how well multiarray backprojection can resolve events closely separated in space and time. To address this, we have created two point sources at different locations and different depths and simulated a propagating rupture by introducing a time interval between the sources. The time interval was set as the horizontal distance between the sources divided by a rupture velocity of $2.5 \mathrm{~km} \mathrm{~s}^{-1}$. The results in Fig. 7 show that the method is capable of resolving such closely spaced point sources. Although we display only two sources we experimented with more sources and the results matched the results shown here.

The recovered sources are elliptically shaped and generally elongated in the northeast-southwest direction due to the higher array stack contribution from the Australian arrays (Figs 7(a) and (b)). In depth the separate sources are also well resolved, again with slight 

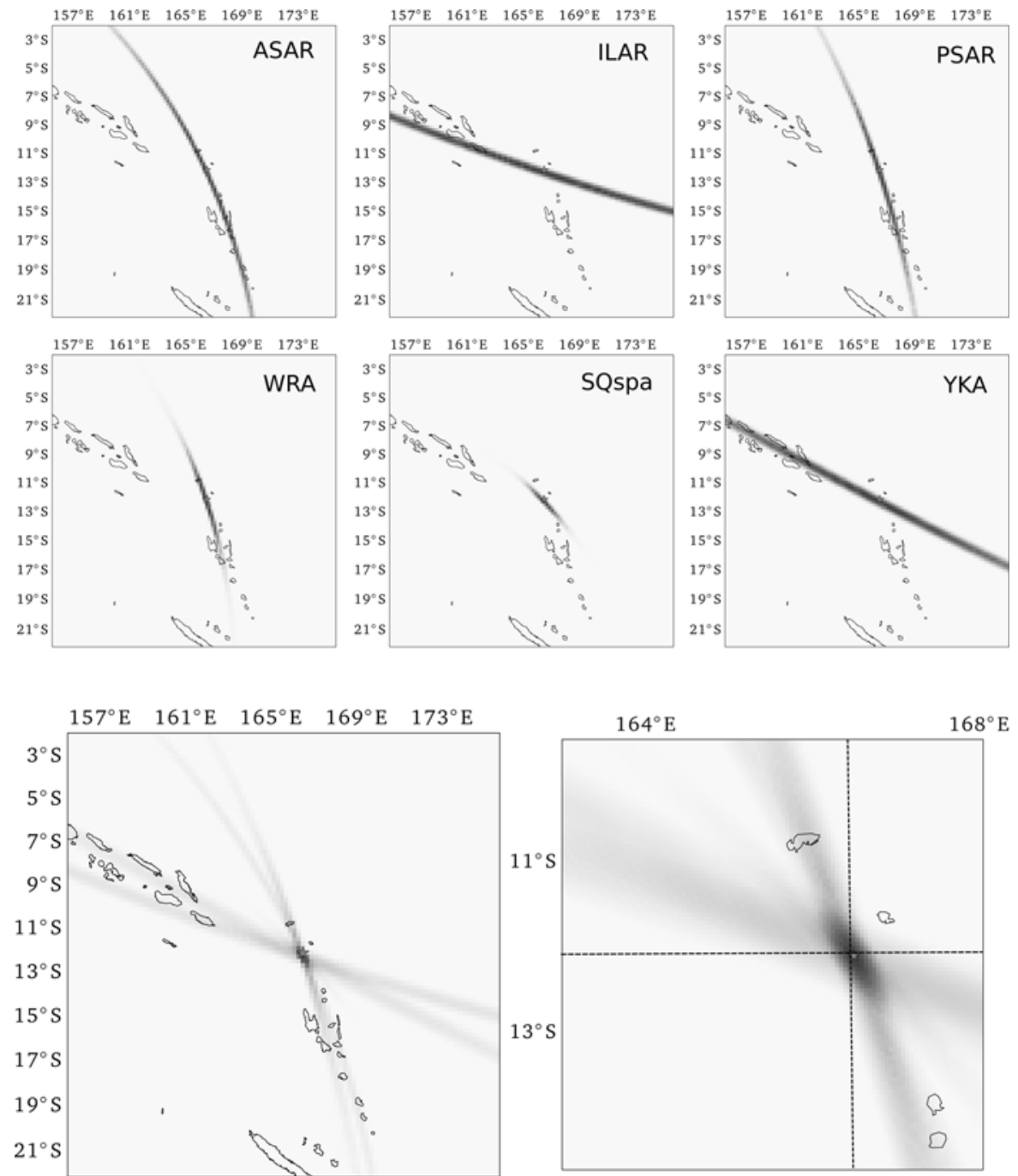

Figure 4. Lateral resolution. Six upper panels show individual array beam-power contributions. Two lower panels show combined beam-power maps with left images showing results calculated using the coarser grid spacing $0.2^{\circ} \times 0.2^{\circ}$ and right panel showing results on the finer grid $0.05^{\circ} \times 0.05^{\circ}$. The beam-power colour scale is linear and varies from off-white (low values) through dark red (high values) and red star marks the true source location. Dashed lines mark the locations of the cross-sections shown in Fig. 5.

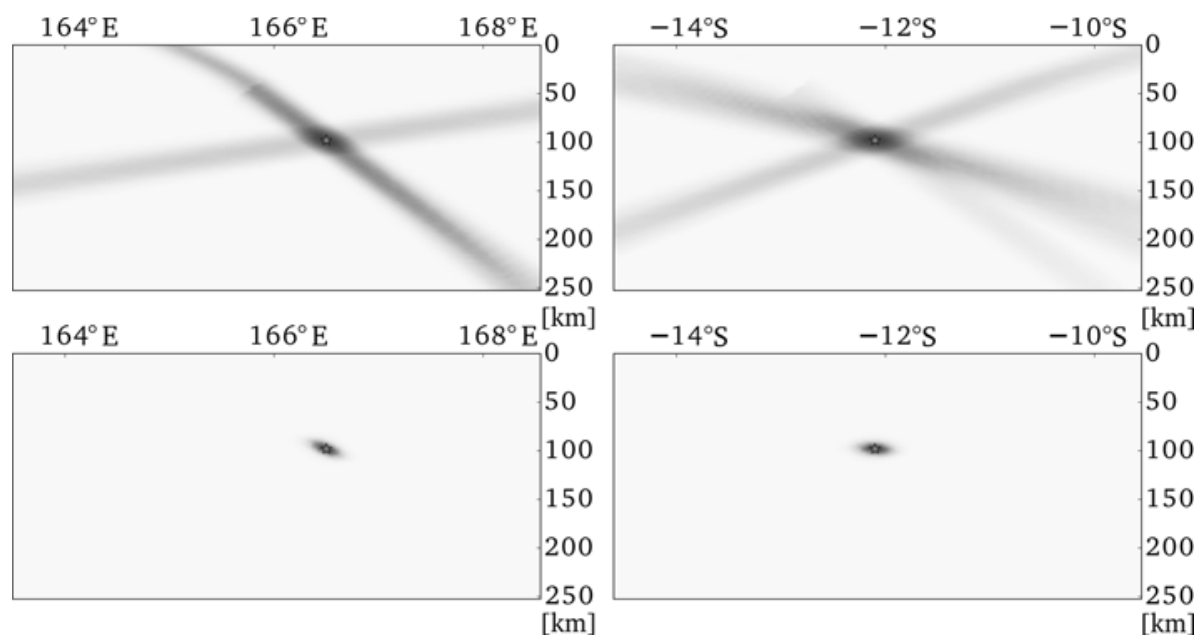

Figure 5. Cross-section of the beam power results in depth using a $0.05^{\circ} \times 2.5 \mathrm{~km}$ grid extending to $250 \mathrm{~km}$. The upper panels show cross-sections at the hypocentral latitude $\left(-12.1^{\circ}\right)$ and longitude $\left(166.5^{\circ}\right)$ when using the summed beam-power maps. Two lower panels are the same cross-sections created using the pointwise grid multiplication of the power contributions from the different arrays. 

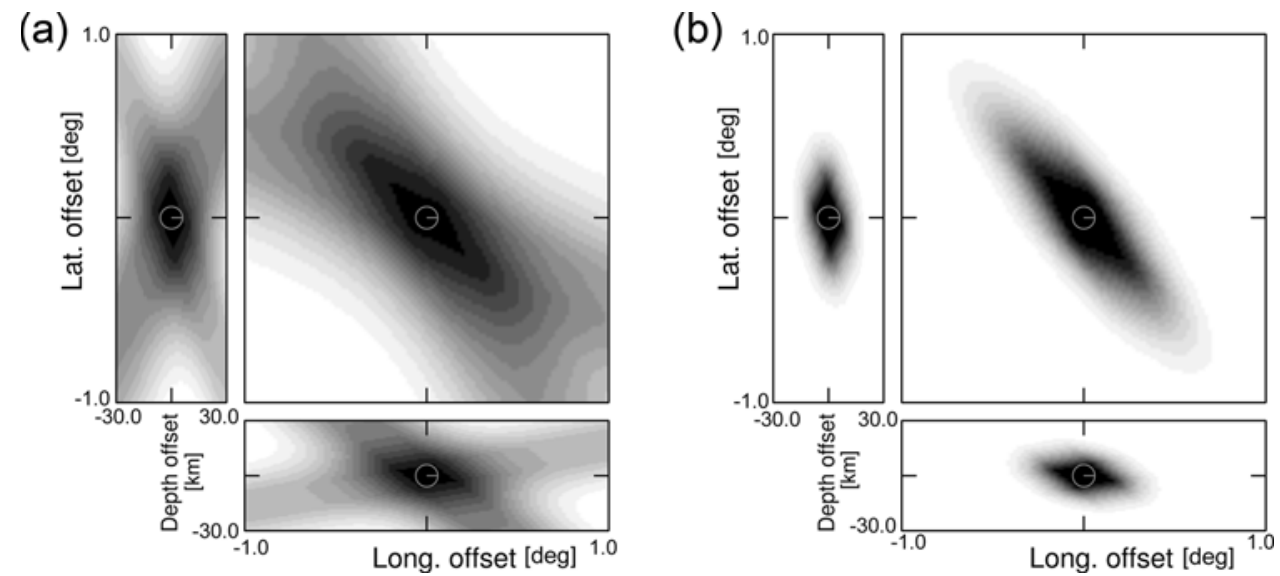

Figure 6. Representation of spatial resolution for the event in Solomon Islands at $100 \mathrm{~km}$ depth using all six arrays (ASAR, PSAR, WRA, SQspa, ILAR and YKA) with three separate cross-sections through the event location. Left panel shows the resolving power when using summation of the individual array contributions and right panel shows resolution when using multiplication (see the text for details).

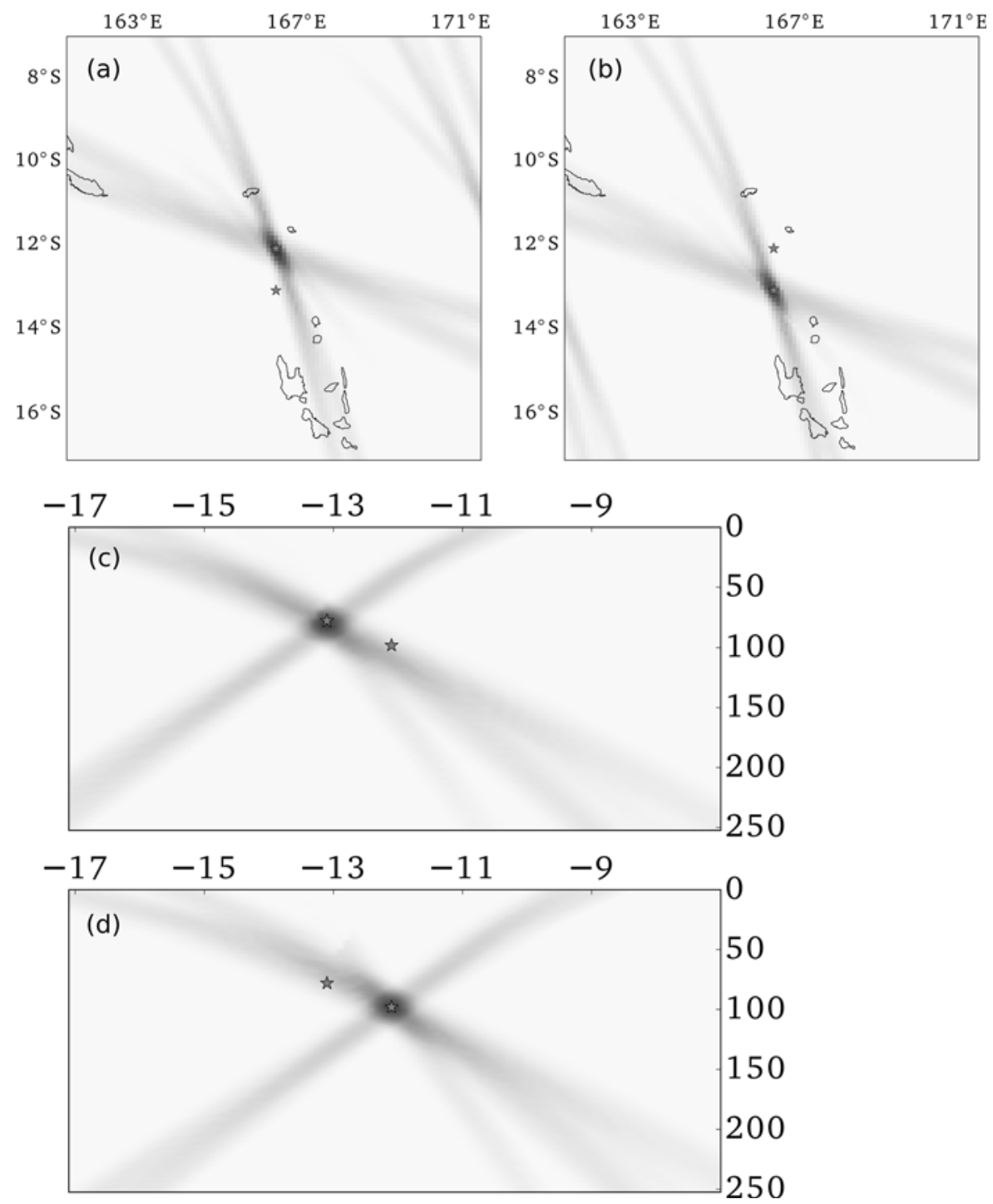

Figure 7. Multiarray beam power results for two sources separated in space and time. The upper panels show map views of the results at the correct source times. The left panel displays the multiarray beam-power mapping at the source time of the first source, whereas in the right panel we show the results for $44.5 \mathrm{~s}$ after the initiation of the first source, that is, at the onset of the second source. The lower two panels illustrate cross-sections at the source longitude $\left(166.5^{\circ}\right.$ - the same for both sources) at the source times, with the upper panel displaying the source with earlier source time. 


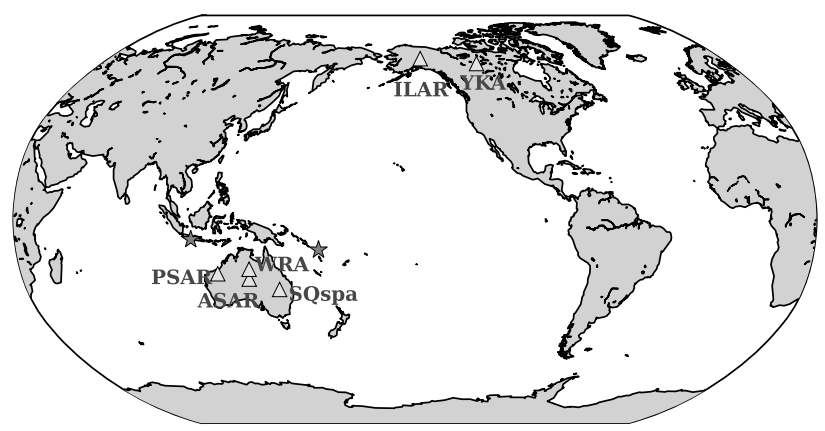

Figure 8. Location of the arrays (blue triangles) and the two events (red stars) used in this study.

smearing in depth due to the higher contribution from the closer arrays. An important contribution to depth resolution comes from the distant arrays. In this simulation we spaced sources to match the area of the highest beam power for Australian arrays. Without the North American arrays (YKA and ILAR) we would not be able to resolve two sources. This goes to show the importance of using at least one array which is separated in azimuth, and possibly also in distance, from the other arrays used in the analysis.

\section{MULTIARRAY PROCESSING AND APPLICATION}

In order to test the multiarray backprojection technique on real data we use recordings of two intermediate-depth earthquakes recorded at six arrays (ASAR, PSAR, WRA, SQspa, ILAR and YKA) each with different configuration and aperture. The configuration of the events and the arrays employed is shown in Fig. 8. Both of the earthquakes are medium sized events to avoid obscuring energy projection to the source location with overly complex source mechanisms. Nevertheless, such multiarray projection is applicable to tracking earthquake source evolution of large earthquakes (Kennett et al. 2014).

In these examples we concentrate on the 3-D spatial resolution of the seismic source for a given origin time. As demonstrated in Section 4.5 we do have time discrimination. We can track the evolution of the emission of energy by using a sliding window in time, tracking along the seismograms at the different arrays, with appropriate relative time delays ( $c f$. Kennett et al. 2014). With the same spatial grid, the slowness components and relative timing remain the same for each grid point, which simplifies the calculations.

We use just the first arriving $\mathrm{P}$ phase in the illustrations, but stacking can be easily extended to use additional phases (e.g. pP or sP) as shown in Kiser et al. (2011). Our multiple array projection procedure broadly follows that described by Roessler et al. (2010). First, an evenly spaced 3-D grid (latitude, longitude and depth) of potential source locations is created. Each array is processed independently, but we use a common, global grid of points so that the arrays can later be combined. For each grid point a beam is formed by stacking the seismograms with appropriate time shifts, and the beam power is calculated from a short tapered window centred on the traveltime to each grid point. The time shifts have two components: the traveltime from each grid point to the reference point of the array, and a slowness-dependent offset-time from each station of the array to the reference point. As described in Section 4, this combined time shift provides good potential control for depth resolution. Before performing the stacking procedure, we apply a bandpass filter to each trace with corners at 0.5 and $4.0 \mathrm{~Hz}$, to extract the higher frequency part of the seismograms. We work with $4 \mathrm{~s}$ time windows tapered with a Kaiser window, with $\alpha$ parameter set to 2 thus providing good tapering of the outer ends while preserving the central section of the window. The $4 \mathrm{~s}$ window employed in the multiarray projection allows for a time interval of $\pm 2 \mathrm{~s}$ around the expected $P$ wave times for the current target. We tested several window sizes $(2,4,6,10$ and $20 \mathrm{~s})$, and have come to the conclusion that for projection of high frequency $P$ waves a $4 \mathrm{~s}$ window sets a good balance between exploiting the frequency content of the signal and the effective resolving power of the method. The type of stacking to create the beam has a significant effect on the result. We use a nonlinear phase-weighted stacking scheme (Schimmel \& Paulssen 1997) which mitigates the effects of anomalous individual waveforms and enhances coherent energy, thus reducing spread of the beam power and effectively enhancing the resolution. In the final step we combine the gridded beam power from all the arrays by pointwise summation or multiplication ( $c f$. Roessler et al. 2010). In order to give each array beam power equal weight we normalize array beam power before summation or multiplication.

We divide the process of multiarray projection into two stages. In the first stage we construct a coarse grid around the target location with dimensions $20^{\circ}$ in latitude, $20^{\circ}$ in longitude and $250 \mathrm{~km}$ in depth. The grid spacing is set to $0.2^{\circ} \times 0.2^{\circ} \times 5 \mathrm{~km}$. After determining the area with the largest stack power, we create much denser grid around that area with grid spacing of $0.05^{\circ} \times 0.05^{\circ} \times 2.5 \mathrm{~km}$. This two-stage approach provides a rapid estimation of the initial source location and gives insight into possible spurious source locations for noisy data.

\subsection{South Solomon Islands event}

The $M_{\mathrm{w}}$ 6.0 Solomon Islands earthquake on 2014 March 27 at 03:49 GMT, occurred at the subduction zone where the Australian Plate descends beneath the Pacific Plate with different convergence directions. The hypocentral depth from the ANSS catalogue (http://www.quake.geo.berkeley.edu/anss/) is $98 \mathrm{~km}$.

As can be seen from the results of the synthetic test shown in Fig. 4, with only Australian based arrays we get reasonable azimuthal control on the source location. Spatial resolution at the hypocentre is quite good in the direction perpendicular to the back-azimuth and significantly elongated in depth along the backazimuthal direction. The two main reasons for depth smearing in the back-azimuth direction are poor azimuthal coverage and, more importantly, the spatial configuration of the arrays. Because the Australian arrays are relatively close to each other and nearly equidistant from the source the separation in slowness is small, which has a strong influence on depth discrimination when stacking the array beams. The control provided by such a restricted set of four arrays is undoubtedly limited, but serves the purpose of fast location of the source area as we do not use a predetermined source location.

In order to achieve good control in both azimuthal and spatial resolution at the hypocentre we need to use all the available arrays. Azimuthal separation helps to narrow the area of overlap of isochrons and hence increases resolution (e.g. Kennett et al. 2014). Further, because the event energy arrives at ILAR and YKA almost vertically the combination with the low angle Australian beams provides an excellent platform for determining depth. We start by calculating beam-power maps on the coarse grid. As can be seen from the results in Figs 9(a)-(c) the source area is well defined and closely coincides with the hypocentral location from the ANSS catalogue. Contributions from each array are shown in Supporting Information Fig. S2. 

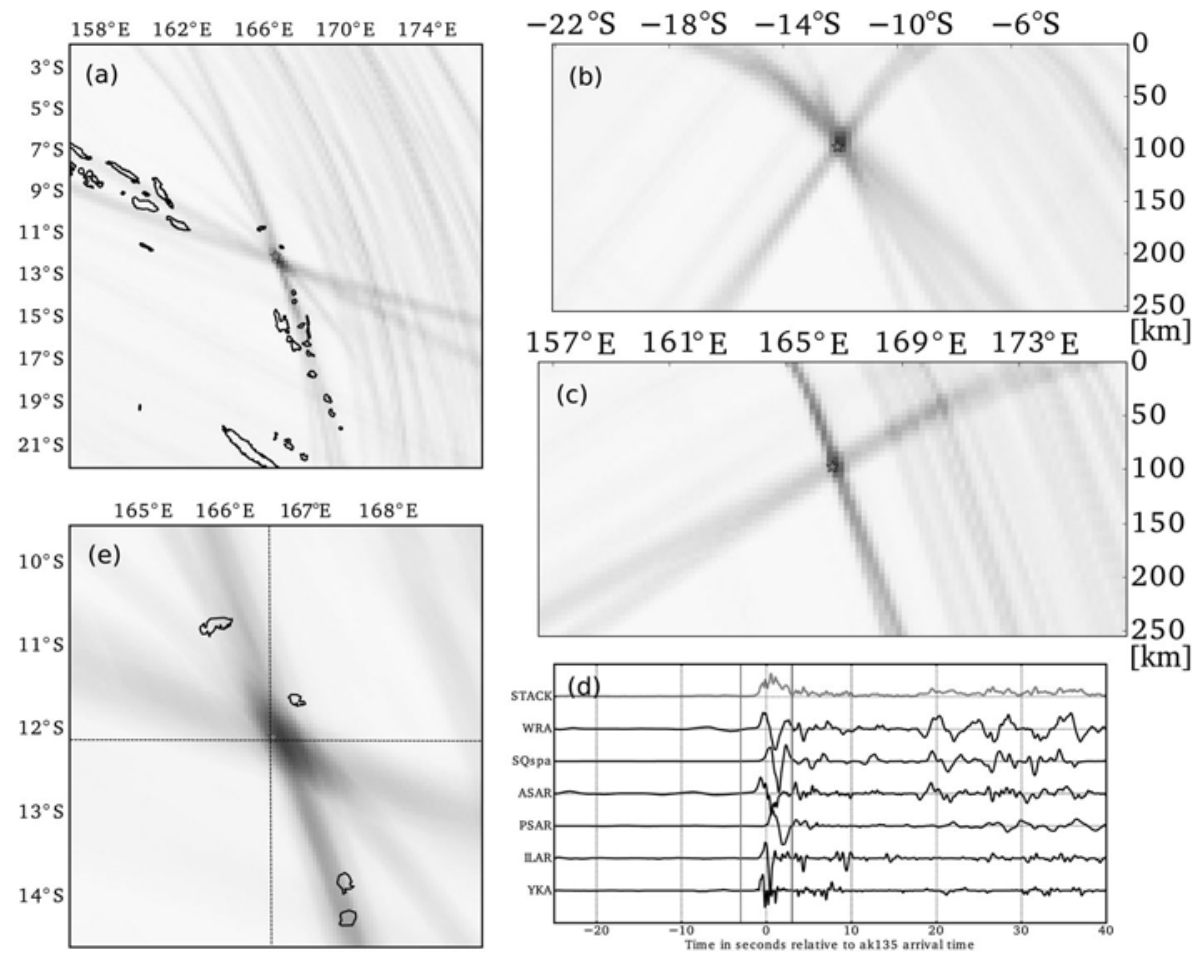

$[\mathrm{km}]$

Figure 9. Illustration of multiarray projection for the Solomon Islands event. The beam-power colour scale is linear and varies from off-white (low values) through dark red (high values). The red star is the location of the hypocentre taken from the ANSS catalogue (http://www.quake.geo.berkeley.edu/anss/). Panels (a)-(c) show multiarray projection results using the coarse grid. While panel (e) shows results using the finer grid spacing. Panels (a) and (e) show a map view of the results at the catalogue hypocentral depth $(98 \mathrm{~km})$, (b) and (c) are cross-sections at the hypocentral latitude $\left(-12.1^{\circ}\right)$ and longitude $\left(166.5^{\circ}\right)$. Panel $(\mathrm{d})$ depicts the phase-weighted stack for each array separately (in black) and a stack of absolute value array stacks (in red), which is proportional to the beam power. The stacks are calculated at the location of the hypocentre from the ANSS catalogue. Red vertical lines indicate the $4 \mathrm{~s}$ window used in the beam-power calculation. Dashed lines in panel (e) mark the locations of the cross-sections shown in panels (b) and (c).

We now move to the second step of the multiarray projection and use all the available arrays on a refined grid in order to achieve best possible resolution. In Fig. 9(e), we display the results of the multiarray projection for the Solomon Islands event computed on a finer grid drawn around the maximum beam-power area on the initial coarse grid. The plot shows one clearly defined maximum, very tightly concentrated about the source location.

In the initial tests the calculated maximum of the beam energy did not coincide precisely with the catalogue source location. The difference is most likely caused by $3-\mathrm{D}$ effects around the source (or receiver), combined with the uneven array distribution, that is, more arrays to one side by comparison with the other. The array stacks in Fig. 10(c) show this effect more clearly. Stacks for the Australian based arrays are centred on the predicted arrival time for ak135, while the stacks from two North American stations show small time offsets. We can make a significant improvement by introducing arrival time corrections based on the work of Nicholson et al. (2004). Their empirical traveltime scheme can be applied readily to any array operating for a period of time and does not depend on an explicit 3-D earth model. Once this style of time correction is implemented the alignment of the different array contributions is much improved (Fig. 10c) and the correspondence between the array-based location and the estimated hypocentre is very close Fig. 10(b). Though we note that the beam-power maximum will represent the point of maximum energy emission in the $4 \mathrm{~s}$ window, and does not have to coincide with the point of energy initiation, the hypocentre.

In Figs 9(a)-(d), we see a set of streaks displaced from the main group. These auxiliary beam-power maxima are connected with the secondary arrivals such as $\mathrm{pP}$ and $\mathrm{sP}$ or crustal scattering on the source or receiver side. These secondary phases have similar slowness to the main P arrival and modest separation in time. As the test point moves away from the true location the relative timing of the array contributions is altered, and the combination when stacking at this grid point can combine a beam from the main $\mathrm{P}$ arrival at one array with secondary arrivals at another. The general rule is for shallower source depth and shorter epicentral distance, the displaced streaks will be stronger and harder to discern from the main P arrival stack. Whereas the streaks from secondary arrivals are easily recognizable, scattering is rather more tricky as forward scattering can produce arrivals coming before main $P$ phase. Usually the energy of these locally scattered arrivals is low and is not present at all the arrays, which in turn means that the beam-power stack of the scattered energy will be small.

We can partially mitigate such unwanted 'side lobes' by an alternative construction of the composite array response. Rather than summing array stacks directly we undertake pointwise grid multiplication of separate array beam powers. This means that at each grid point we calculate beam-power contribution from each of the arrays separately and then multiply array beam-power contributions from all the arrays to get the beam-power value at that grid point. The difference can be seen in Figs 5 and 12 where we apply the multiplication procedure to the beam results with time corrections. The array projection method can be extended to make explicit use of additional phases (pP, sP, etc.), and to include differential weighting of the array power stacks when summing. We have experimented with such modifications, and have found that the energy-projection results are very robust and except for very deep events there is not likely to be much benefit from isolating multiple phase windows. 

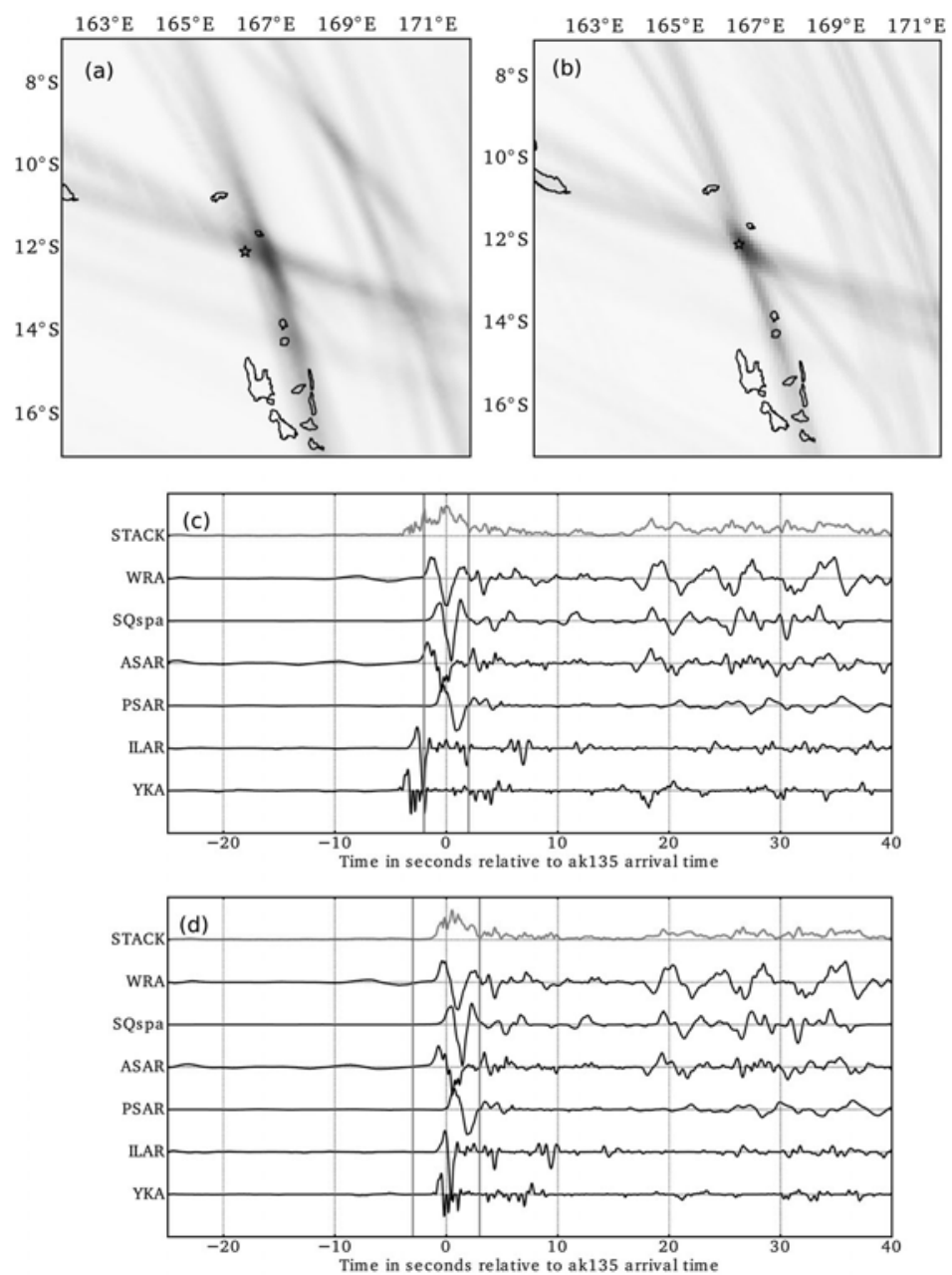

Figure 10. Comparison of array projection for the Solomon Islands event with and without time corrections based on empirical 3-D traveltimes. The red star is the location of the hypocentre taken from the ANSS catalogue. The upper panels show map view of the results at the catalogue hypocentral depth (98 km) without (a) and with (b) time corrections. The lower panels depict the phase-weighted stack for each array separately (in black) and a stack of absolute value array stacks (in red), Stacking without time corrections is displayed in panel (c) and with time corrections in panel (d).

\subsection{Central Java event}

Our second example is the $M_{\mathrm{W}} 6.1$ Central Java earthquake which happened on 2014 January 25 at 05:14 GMT. The ANSS catalogue gives the location just offshore central Java at an estimated depth of $66 \mathrm{~km}$. We chose this event because of its good location in respect to the array configuration in Australia. Furthermore, we wanted to see how the multiarray method behaves when the source is relatively shallow with several different phases arriving close to each other, and where scattering around the source can influence the result.

We start by calculating average beam power on a coarse grid encompassing a large area around Java. Contributions from each array separately are shown in Supporting Information Fig. S3. We apply the time corrections from Nicholson et al. (2004) to better constrain maximum beam power. In a similar way to the Solomon Islands event there is arcuate smearing in the spatial resolution, although now somewhat higher because of the higher contribution from the Australian arrays (Fig. 11). The area of high beam power is also less well constrained in depth, which shows the importance of good distance coverage.

When we compare the Java event with the Solomons event, we see much more scattered energy in the beam-power pattern. There is significant smearing in spatial and depth resolution which is connected with scattering and several strong phases arriving close to each other. Nevertheless, the multiarray approach is able to locate the source location as shown in Figs 11(a)-(c). Multiarray projection on a denser grid is displayed in Fig. 11(e). In cases when there is a lot of artefacts it is better to use pointwise multiplication to combine the array contributions. By using multiplication we are able to extract the areas with the highest values and subdue the side lobes. As shown in Fig. 12 when we use multiplication the source area is tightly constrained and artefacts are removed. The maximum beam-power area is slightly shifted from the catalogue hypocentre location due to the strong phase arriving at Australian arrays close to the main P phase (see Fig. 11(d)). In spite of all these disturbances when using the multiarray projection and multiplying array power maps we are able to get excellent estimate of the source location.

\section{DISCUSSION AND CONCLUSIONS}

In the classic backprojection procedure the lateral resolution is controlled by the distance and azimuthal coverage (Kiser \& Ishii 2012), but source depth is relatively poorly resolved. By using several small-medium sized arrays we are able to constrain depth by 

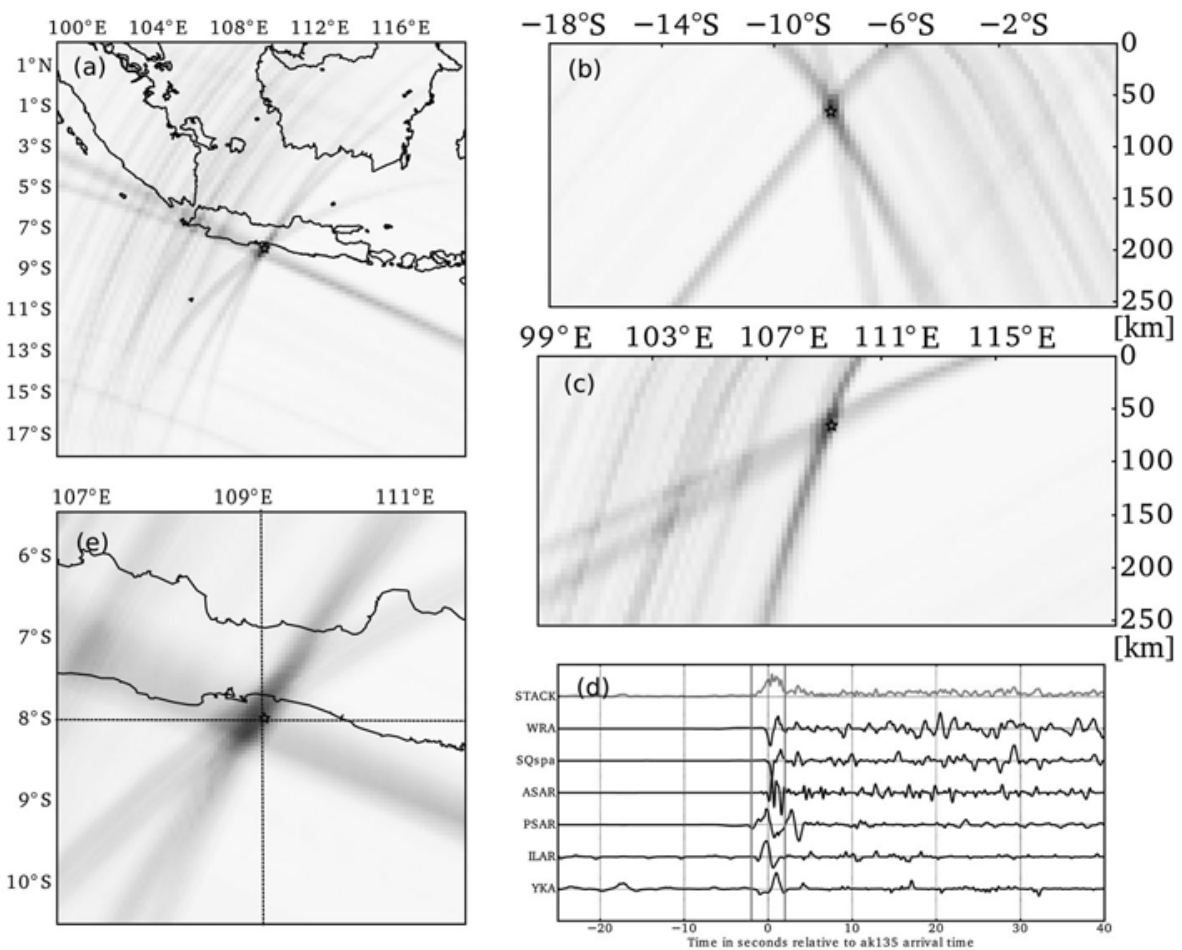

Figure 11. Illustration of multiarray projection for the Central Java event. The beam-power colour scale is linear and varies from off-white (low values) through dark red (high values). The red star is the location of the hypocentre taken from the ANSS catalogue (http://www.quake.geo.berkeley.edu/anss/). Panels (a)-(c) show multiarray projection results using the coarse grid. While panel (e) shows results using the finer grid spacing. Panels (a) and (e) show a map view of the results at the catalogue hypocentral depth $(66 \mathrm{~km})$, (b) and (c) are cross-sections at the hypocentral longitude $\left(109.26^{\circ}\right)$ and latitude $\left(-7.98^{\circ}\right)$. Panel (d) depicts the phase-weighted stack for each array separately (in black) and a stack of absolute value array stacks (in red), which is proportional to the beam power. The stacks are calculated at the location of the hypocentre from the ANSS catalogue. Red vertical lines indicate the $4 \mathrm{~s}$ window used in the beam-power calculation. Dashed lines in panel (e) mark the locations of the cross-sections shown in panels (b) and (c).

maximizing beam-stacks across the target area. We have shown that by applying simple traveltime and slowness constraints it is possible to construct a simple measure of spatial resolution and also to quickly locate the source area.

One of the advantages of the multiarray method is that we do not have to correct for lateral offsets, as the rays impinging at the array will be coming from the approximately same area and thus it is a good approximation to assume plane wave fronts. We do not have to impose a fixed hypocentre location, but can instead find the point of maximum energy emission within a broadly defined target volume through a grid search process. One of the benefits is that this procedure can be applied to any point inside the Earth, for example, we can locate deep mantle scatterers with multiarray beaming by simply using appropriate time and slowness constrains while having benefit of enhanced SNR at high frequencies.

Using many small to medium aperture arrays has a number of advantages. We can exploit existing arrays using standard planewave processing. Further such modest-size arrays can be readily deployed using portable instruments with geometries constructed to maximize SNR (e.g. Kennett et al. 2015). For source/scattering studies, a couple of well-placed arrays can easily replace much larger arrays with a hundred or more elements.

The multiarray beamforming process can be combined with the tracking of energy release in time for large earthquakes as in Kennett et al. (2014). Once the primary source location grid is established the centre of time window is incremented and the new evocentre corresponding to maximum energy emission tracked in space. The search grid can be adaptive moving with the evolution of the event energy.

\subsection{Large arrays}

Large arrays can also be used in a multiarray approach provided the nature of the recorded wavefield is taken into account. For a large array there will be curvature of a phase wave front across the array, and then phasing can be achieved by using a non-plane wave reference field. For example the theoretical arrival times calculated for a reference earth model, such as ak135 (Kennett et al. 1995), can be used to align the seismograms before stacking (e.g. Rawlinson \& Kennett 2004). Note, that just as in the full multiarray case, this requires the immediate specification of a target location to which the beaming is directed.

If station density is high enough it may be advantageous to break a larger array into a number of smaller sub-arrays for which the plane wave assumption is adequate. Beams can then be calculated for each of the sub-arrays and combined to enhance the overall response using the procedures described above.

\subsection{Multiarray monitoring}

The simultaneous use of multiple arrays opens possibilities for realtime analysis. In the simplest approach, independent beamforming at different arrays can be used to triangulate on a broad area of likely energy emission. This region can then be used to initiate a detailed 

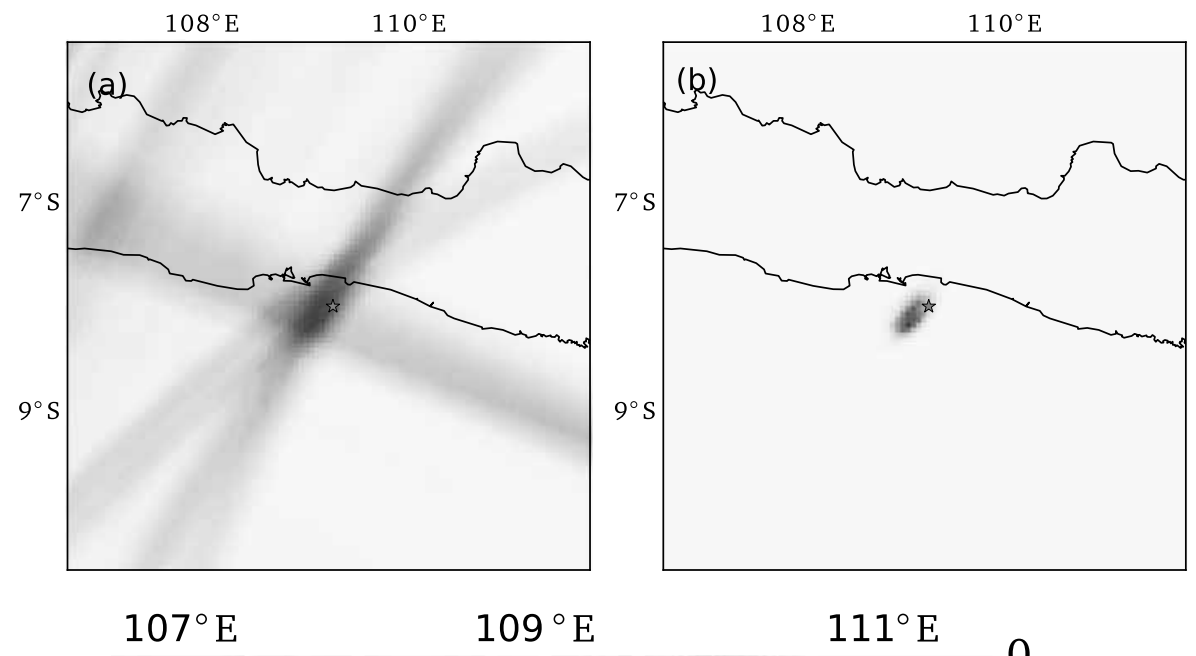

$111^{\circ} \mathrm{E}$

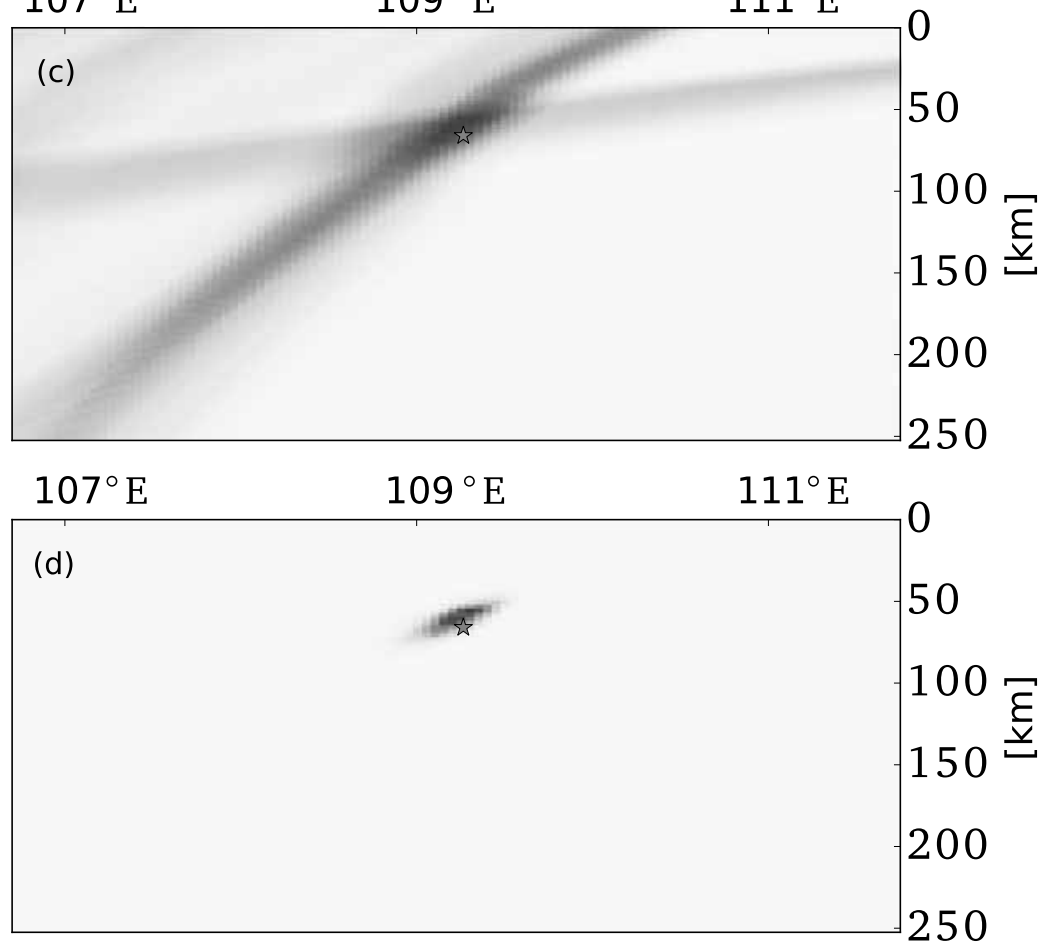

Figure 12. Comparison of summed versus multiplied beam-power maps for the Java event. The two upper panels show the lateral resolution when (a) using summed beam-power maps, and (b) when using multiplication. Panels (c) and (d) compare the depth resolution for summed and multiplied beam-power maps.

grid search taking into account the propagation time delays to the different arrays.

An alternative when working with a fixed complement of arrays is to establish a preset template of likely source locations, with regular monitoring of the effective multiarray beam power at each target as a function of time. When the multiarray beam exceeds a threshold indicative of a likely event, then a full 3-D search is initiated in the neighbourhood using the recently received seismograms. The trigger then provides a direct indicator of the potential origin time of the event. Such an approach builds on the threshold monitoring scheme of Kværna et al. (2002a,b) to initiate an active search to improve energy localization as a supplement to other ways of monitoring. When combined with tracking the location of maximum beam power in space and time (cf. Kennett et al. 2014), the trajectory of the evocentres can provide direct information on the evolution of an event and hence, for example, its tsunami potential.

\section{ACKNOWLEDGEMENTS}

This work has been supported in part by ARC Discovery Grant DP130101473 and Croatian Science Foundation Project IP-201409-9666.

A number of members of RSES, ANU have helped with the temporary array in southern Queensland (SQspa), and in particular Qi Li and Geoff Luton for their efforts in the field and Armando Arcidiaco for his efforts in the field and the work on the assembly of the array data from the component stations.

\section{REFERENCES}

Bowers, D. \& Selby, N.D., 2009. Forensic seismology and the Comprehensive Nuclear-Test-Ban Treaty, Annu. Rev. Earth planet. Sci., 37, 209-286. Douglas, A., 2013. Forensic Seismology and Nuclear Test Bans, Cambridge Univ. Press. 
Gal, M., Reading, A.M., Ellingsen, S.P., Koper, K.D., Gibbons, S.J. \& Nasholm, S.P., 2014. Improved implementation of the fk and Capon methods for array analysis of seismic noise, Geophys. J. Int., 198, 1045-1054.

Kennett, B.L.N., 2002. The Seismic Wavefield II: Interpretation of Seismograms on Regional and Global scales, Cambridge Univ. Press.

Kennett, B.L.N., Engdahl, E.R. \& Buland, R., 1995. Constraints on seismic velocities in the Earth from travel times, Geophys. J. Int., 122, 108-124.

Kennett, B.L.N., Gorbatov, A. \& Spiliopoulos, S., 2014. Tracking earthquake source evolution in 3-D, Geophys. J. Int., 198, 867-879.

Kennett, B.L.N., Stipčević, J. \& Gorbatov, A., 2015. Spiral arm seismic arrays, Bull. seism. Soc. Am., 105, 2109-2116.

Kiser, E. \& Ishii, M., 2012. Combining seismic arrays to image the highfrequency characteristics of large earthquakes, Geophys. J. Int., 188, 1117-1128.

Kiser, E., Ishii, M., Langmuir, C.H., Shearer, P.M. \& Hirose, H., 2011. Insights into the mechanism of intermediate-depth earthquakes from source properties as imaged by back projection of multiple seismic phases, $J$. Geophys. Res, 116, B06310, doi:10.1029/2010JB007831.

Kværna, T., Ringdal, F., Schweitzer, J. \& Taylor, L., 2002a. Optimized seismic threshold monitoring-Part 1: Regional processing, Pure appl. Geophys., 159, 969-987.

Kværna, T., Ringdal, F., Schweitzer, J. \& Taylor, L., 2002b. Optimized seismic threshold monitoring - Part 2: Teleseismic processing, Pure appl. Geophys., 159, 989-1024.

Nicholson, T., Sambridge, M. \& Gudmundsson, Ó., 2004. Threedimensional empirical traveltimes: construction and applications, Geophys. J. Int., 156(2), 307-328.

Rawlinson, N. \& Kennett, B.L.N., 2004. Rapid estimation of relative and absolute delay times across a network by adaptive stacking, Geophys. J. Int., 157, 332-340.

Ricker, N., 1953. The form and laws of propagation of seismic wavelets, Geophysics, 18(1), 10-40.

Roessler, D., Krueger, F., Ohrnberger, M. \& Ehlert, L., 2010. Rapid characterisation of large earthquakes by multiple broadband arrays, Nat. Hazards Earth Syst. Sci., 10, 923-992.

Rost, S. \& Thomas, C., 2002. Array seismology: methods and applications, Rev. Geophys., 40(3), 1008, 2-1-2-27.

Schimmel, M. \& Paulssen, H., 1997. Noise reduction and detection of weak, coherent signals through phase-weighted stacks, Geophys. J. Int., 130(2), 497-505.
Simons, M. et al., 2011. The 2011 magnitude 9.0 Tohoku-Oki earthquake: mosaicking the megathrust from seconds to centuries, Science, 332, 1421-1425.

Ye, L., Lay, T., Kanamori, H. \& Koper, K.D., 2013. Energy release of the $2013 M_{\mathrm{W}} 8.3$ Sea of Okhotsk earthquake and deep slab stress heterogeneity, Science, 341, 1380-1384.

\section{SUPPORTING INFORMATION}

Supplementary data are available at GJIRAS online.

Figure S1. The array responses in slowness space for the six arrays (ASAR, ILAR, PSAR, WRA, SQspa and YKA) used in this study. The relative power in decibels is plotted as a function of horizontal components of the slowness vector over the interval $(-1.0$ to $1.0 \mathrm{~km}^{-1}$ ). The responses are computed for a monochromatic $(1 \mathrm{~Hz})$ wave entering the array domain vertically from below, darker colours indicate higher power.

Figure S2. The panels show the individual array beam-power contributions for the Solomon Islands event (2014 March 27) using the coarse grid $\left(0.2^{\circ} \times 0.2^{\circ}\right)$ The beam-power colour scale is linear and varies from off-white (low values) to dark red (high values) and the central yellow star marks the true source location.

Figure S3. The panels show the individual array beam powercontributions for the shallow event in Central Java (2014 January $25)$ using the coarse grid $\left(0.2^{\circ} \times 0.2^{\circ}\right)$. The beam-power colour scale is linear and varies from off-white (low values) to dark red (high values) and the central yellow star marks the true source location.

Table S1. Minimal and maximal distance between stations in the six arrays used.

Please note: Oxford University Press is not responsible for the content or functionality of any supporting materials supplied by the authors. Any queries (other than missing material) should be directed to the corresponding author for the paper. 